\title{
La formation des enseignants au Chili : est-elle une mimêsis de la politique (trans)nationale?
}

\section{Javier Núñez-Moscoso \\ Universidad de 0'Higgins (ICEd-UOH) (Chili)}

\author{
Teacher training in Chile: A (trans)national mimesis policy?
}

doi: $10.18162 / f p .2021 .657$

\section{ésumé}

Cette contribution propose un regard critique sur la manière dont le Chili adopte et incarne (mimêsis) certaines préconisations d'organismes internationaux en matière de politiques éducatives. L'argument central est déployé autour de la description documentée de la façon dont certaines orientations préconisées par les organismes internationaux ont été intégrées dans le champ de la formation des enseignants au Chili, au travers des réformes et dispositifs à l'œuvre dans les dernières décennies et plus récemment (Núnez-Moscoso et Flores Gonzalez, 2017). L'article se termine par une réflexion sur les potentialités et les risques de ces transformations pour la profession enseignante.

Mots-clés

Chili, Formation des enseignants, globalisation, mimèsis, politiques éducatives.

Abstract

This contribution presents a critical look at the way Chile adopts and embodies (mimesis) some of the recommendations of international organizations that affect educational policies. The central argument is deployed around the documented description of how certain orientations have been integrated into the field of teacher training in the country, within the reforms and changes of the last decades but also the most recent ones (NúñezMoscoso et Flores Gonzalez, 2017). The article ends with a description of the potentialities and risks that these transformations may have for the teaching profession.

Keywords

Chile, Teacher training, globalization, mimesis, educational policy.

\section{Introduction}

Paris, France, siège de l'United Nations Educational, Scientific and Cultural Organization (UNESCO) et de l'Organisation for Economic Co-operation and Development (OECD). New York, États-Unis, siège de la Banque Mondiale et de la Banque Ibéroaméricaine de Développement (BID). Ces organismes, dans des villes si lointaines, peuvent-ils avoir une influence sur l'«Escuela F-50 de Villa Las Estrellas ", l'une des écoles la plus australe de la planète (Antarctique chilienne)? Absolument.

Depuis plusieurs décennies, certains organismes internationaux formulent des préconisations sur la base d'études et expertises, afin d'induire des changements dans les politiques éducatives de différents pays, notamment auprès des nations en voie de développement (Canan, 2017). Le Chili s'est montré particulièrement sensible à ces directives, le champ de la profession enseignante ayant lui aussi fait l'objet d'importantes transformations portant sur la formation initiale des enseignants, l'évaluation et l'évolution de la carrière enseignante. Dans notre posture interprétative, ces processus de transformations à l'œuvre sous l'impulsion de grands opérateurs internationaux engagent, dans le cas du Chili, à la fois un emprunt des politiques éducatives, mais aussi un état de «transe », d'enchantement acritique envers les préconisations émanant d'organismes internationaux que nous appellerons « mimêsis » (Bozal, 1987).

Dans la continuité d'une réflexion menée autour du «paradigme chilien » en matière de politiques de formation du professorat (Núñez-Moscoso et Flores Gonzalez, 2017)', cette contribution se propose de revenir sur la question à travers une perspective essentiellement critique. Pour ce faire, cet article présentera d'abord l'hypothèse heuristique de la «mimêsis » des politiques éducatives 
chiliennes vis-à-vis des préconisations internationales, c'est-à-dire l'adoption et l'incarnation exacerbées de certaines orientations en matière de politiques éducatives dans les transformations à l'œuvre dans le système éducatif chilien, et plus particulièrement en ce qui a trait à la formation des enseignants. À travers ce prisme, nous décrirons ainsi la percée concrète des préconisations des organismes internationaux sur le plan des politiques éducatives dans le contexte chilien. Postérieurement, nous présenterons le contexte de la formation initiale des enseignants au Chili, en mettant par ailleurs l'accent sur les transformations récentes portées par la loi 20.903 du 4 mars 2016 ou ley de la carrera docente (loi de la carrière enseignante). Finalement, nous conclurons par quelques pistes de réflexion sur les potentialités, les risques et les tensions liés à l'influence internationale dans la transformation de la formation des enseignants, ainsi que ses potentielles conséquences pour la profession enseignante.

\section{« Mimêsis » et politiques en formation des enseignants : une hypothèse heuristique}

Souvent traduite par «imitation" ou «reproduction ", la notion de «mimêsis » dans la Grèce ancienne fait, selon Gutiérrez Canales (2016), plutôt référence à l'idée d'« incarnation ». Bozal (1987, p. 70) est clair à ce propos : "Mimêsis" dérive de "mimos" et "mimeisthai", des termes qui se réfèrent originalement au changement de personnalité que certains fidèles expérimentaient pendant certains rituels, lorsqu'en eux s'incarnaient des êtres de nature divine ou animale, ou des êtres d'un autre temps. "Mimeisthai" n'est pas autant imiter comme représenter, incarner un être éloigné de soi-même ».

Ainsi, la notion de "mimêsis » fait référence à : 1) l'adoption enchantée d'autres personnalités, éloignées de nous, plaçant l'individu dans une posture de fidélisation acritique ou, dans le meilleur des cas, faiblement critique, 2) l'incarnation exacerbée, de caractère symbolique et cérémonial, propre des liturgies qui cristallisent les croyances religieuses. Cette idée nous semble dès lors particulièrement féconde pour comprendre la percée des préconisations de certains organismes internationaux au Chili.

La « mimêsis » sera donc employée comme hypothèse heuristique, c'est-à-dire comme idée directrice indépendamment de sa valeur de vérité, et qui représente un point de vue, un prisme de lecture (Marcel, 1928) pour appréhender les politiques éducatives liées à la formation des enseignants. De la sorte, cet article défendra l'idée selon laquelle le Chili intègre dans ses politiques éducatives, et de manière particulièrement prégnante, des préconisations venant d'organismes internationaux, à travers leur adoption et leur incarnation profonde. Comme nous le verrons, ce phénomène met en évidence l'importante influence (trans)nationale de la formation du professorat au Chili.

\section{Politiques éducatives : une mouvance transnationale séduisante}

Dans les dernières décennies, la gouvernance des systèmes éducatifs s'est vue transformée : les agendas des pays se sont " globalisés ", ayant, par conséquent, tendance à pointer et assumer les mêmes défis et, souvent, à employer les mêmes mécanismes de résolution de leurs difficultés éducatives, avec au premier chef une place de choix à l'évaluation de l'action éducative (Verger et Parcerisa, 2018). Pour Verger et Parcerisa (2018), les raisons qui expliquent ce phénomène sont liées à la diffusion de politiques éducatives globales, et à l'expansion de politiques basées sur le « new public management », aux avancées techniques et méthodologiques dans le champ de l'évaluation éducative, à l'émergence d'une industrie 
éducative dont l'activité se développe autour des épreuves d'apprentissage, et aux motivations de nature économique pour l'amélioration des résultats d'apprentissage.

Dans la percée de l'influence « internationale $»^{2}$ en Amérique latine, il est possible d'identifier un groupe transnational d'organismes qui favorisent la diffusion d'un ensemble d'orientations déterminées et qui ne se limitent pas à des aspects «techniques » ou d'aide à la décision politique. Bien que l'influence « internationale » soit présente depuis longtemps dans la région³ ${ }^{3}$ pour Beech (2007), l'UNESCO, la Banque mondiale et l'OCDE ont été à l'origine de profondes réformes menées en Amérique latine depuis plus de 30 ans. Précisément, selon cet auteur, ces agences et organismes internationaux ont préconisé, dès la fin des années 80-90, un modèle universel d'éducation basé sur des orientations et principes clefs (Beech, 2005):

- Décentralisation/autonomie scolaire;

- Éducation permanente;

- Curriculum centralisé sur la base de compétences (communication, créativité, flexibilité, apprendre à apprendre, travail en équipe, résolution de problèmes);

- Systèmes centralisés d'évaluation;

- Professionnalisation enseignante.

En reprenant la distinction de Ball (1993) de la politique comme un «texte », c'est-à-dire assujettie à des interprétations par les agents chargés de sa mise en œuvre, et comme un " discours ", autrement dit entendue comme un cadre régulateur créant un agenda éducatif et réduisant le champ interprétatif de ce qui est « acceptable », Beech (2007) défend une hypothèse intéressante. L'auteur soutient l'idée selon laquelle, entre l'abstraction globale et l'appropriation locale, le Chili, comme l'Argentine et le Brésil, a adopté une forte orientation venue «d'ailleurs ». Dans ce sens, « les propositions des agences internationales ont collaboré à modifier le cadre discursif dans lequel se sont pensées les réformes curriculaires de ces pays, mais dans ce cadre il y a eu des interprétations différentes dans chacun des pays analysés " (ibid., p. 162). Quelle a été l'interprétation du Chili au sujet des propositions des agences internationales? Comme nous le montrerons avec le cas de la formation des enseignants, l'interprétation chilienne en a été une d'adoption et d'incarnation profonde de ces préconisations.

Pour Pronko (2000), l'intérêt des organismes internationaux pour l'éducation se cristallise dans une double stratégie : la création d'un pilier pour la politique sociale et d'un pilier pour la politique économique, tous les deux centrés sur l'amélioration de la productivité. Précisément, Oreja Cerruti et Vior (2016) pointent le financent de projets très concrets pour donner forme à cette stratégie : en 2008, le BID a alloué 56.500 USD pour développer les technologies de l'information et de la communication dans l'éducation au Chili; en 2011, le BID a par ailleurs affecté 150.000 USD pour l'insertion des élèves en décrochage scolaire; en 2015, le BID a financé au Chili un programme d'éducation technique et professionnel à la hauteur de 90 millions USD. Sans que ces financements ne touchent directement la formation des enseignants, il semble néanmoins raisonnable d'affirmer l'influence des grands organismes transnationaux sur les politiques éducatives au Chili, avec un intérêt qui se déploie tout à la fois sur le plan symbolique, discursif, financier, et participe de la création d'un agenda éducatif. 
Est-il dès lors possible d'identifier l'écho de cette influence des organismes internationaux dans les politiques de formation des enseignants, et peut-on identifier les deux composantes de la mimêsis, c'est-à-dire l'adoption enchantée et l'incarnation exacerbée?

\section{La formation des enseignants au Chili : contexte et influence internationale}

Cox et Gysling (2009) décrivent l'évolution de la formation des enseignants au Chili au carrefour de trois aspects : le contexte sociohistorique, les savoirs enseignés, et les institutions responsables de la formation des enseignants. Selon ces auteurs, en effet, on peut identifier 4 grands changements qui marquent l'évolution de la formation des enseignants : 1) entre les années 1940 et 1960, le passage d'une formation culturelle à une formation centrée sur les enseignements pédagogiques ou professionnels;2) dans les années 1950, la naissance de la formation pour les enseignants du secondaire marquée par la séparation des instances de production des savoirs disciplinaires (au sein des disciplines universitaires, savantes et érudites) et la reproduction de ceux-ci dans l'enseignement où les enseignants ne participent au développement des savoirs, mais à leur transmission;3) vers les années 1960, l'hégémonie et hiérarchie «supérieure » des savoirs professionnels de caractère théorique versus les savoirs de la pratique moins essentiels, accompagnés par la constitution des Sciences de l'éducation; 4) en début des années 1980, le passage d'une formation des enseignants institutionnalisée, dispensée par un nombre réduit de centres de formation et homogène du point de vue des programmes d'étude, à une autre formation décentralisée et très hétérogène en matière de contenus de formation et d'instances responsables de la formation des enseignants.

Ce dernier changement introduit pendant la dictature militaire (1973-1990) engage la dérégulation de la formation des enseignants, allant de pair avec une dérégulation du système éducatif dans son ensemble. À travers la Constitution de la République de 1980 et de la loi LOCE (Ley Orgánica Constitucional de Enseñanza), le rôle de l'État chilien dans la formation des enseignants (et plus largement dans la gestion de l'éducation) a été fortement revu au profit d'un système reposant sur la « liberté d'enseignement », impliquant la possibilité par divers organismes de proposer des formations à l'enseignement dans un cadre législatif très souple (Oliva, 2008; Valenzuela, Labarrera et Rodriguez, 2008), favorisant par ailleurs l'entrée d'investisseurs privés dans le champ éducatif, et permettant dès lors la création d'institutions éducatives du primaire, secondaire et universitaire (ces dernières responsables de la formation des enseignants) à but lucratif. La formation des enseignants, rattachée auparavant à des structures spécialisées (Escuelas Normalistas ou Institutos Pedagógicos), passe sous la tutelle décentralisée d'un grand nombre d'organismes de formation supérieure (universités privées et publiques, instituts professionnels privés), avec des curriculums très variés et faiblement régulés (Cox et Gysling, 2009).

Plus récemment, bien que la loi LGE (Ley General de Educacion, septembre 2009) remplace l'antérieure (LOCE) en améliorant certains aspects du système, elle conserve certaines limites, par exemple rendre possible l'exercice de l'enseignement dans le milieu secondaire à des professionnels non enseignants. De même, cette loi ne se prononce pas de manière claire sur les conditions de travail des enseignants ou sur la formation initiale, et n'apporte pas de changements substantiels au système de mesures de la qualité de l'éducation. 
Les changements intervenus dans les années 1980 viennent ainsi modifier le cadre et le modèle de formation des enseignants (organisation, curriculums et instances/organismes de formation des enseignants), configurent l'ensemble du système éducatif, et le contexte de travail des enseignants. Le système économique de libre marché, installé formellement dans le pays dès les années 1980, retrouve dès lors son équivalent dans la formation des enseignants sous la logique de développement $\mathrm{du}$ « capital humain » avec comme conséquence la soumission de la formation des enseignants à la rentabilité, où la performance est visée par les processus de professionnalisation. Une évidence de ce phénomène est l'adoption dans le pays d'un modèle de formation par compétences, héritier des expériences menées aux États-Unis, au Canada, en Angleterre et en Australie dans les années 1980 pour améliorer la compétitivité et productivité des travailleurs (Barraza, 2007). Très vite installé dans la formation technique et professionnelle, le modèle par compétences s'est répandu à la formation des élèves et des enseignants, devenant une véritable "vogue ", pour reprendre les termes de Tardif et Desbiens (2014), en Amérique du Nord et en Europe, pour ensuite arriver en Amérique latine. Selon Beech (2005, 2007), les organismes internationaux ont fortement influencé la diffusion du modèle par compétences.

Au Chili, en 1998 est créé le programme MECESUP (Mejoramiento de la Calidad y Equidad de la Educación/Amélioration de la Qualité et Équité de l'Éducation), initiative qui introduit plusieurs changements majeurs, dont «[1]a mise en place au niveau national d'une nouvelle architecture curriculaire pour l'éducation supérieure basée dans les résultats d'apprentissage et la démonstration de compétences » (Cancino Cancino et Iturra Herrera, 2018, p. 329). Plus précisément, dans l'ensemble des projets MECESUP :

[...] l'on a abordé le renouvellement curriculaire sur la base des compétences et des acquis d'apprentissage en considérant de manière plus complète et plus réfléchie la définition des profils des diplômés; les compétences spécifiques et génériques, compte tenu de l'environnement externe; les tendances internationales, y compris les questions sensibles telles que la durée des programmes diplômants; les différents groupes d'intérêt ont été pris en compte, tels que les universitaires, les diplômés, les employeurs, les associations professionnelles et les étudiants. La méthodologie du projet Tuning a également été prise en compte; les avancées du système de crédits transférables (SCT-Chili), avec des mesures de la charge de travail réelle des étudiants; entre autres. (Fernández, 2008, p. 138) ${ }^{4}$

Cet extrait explicite que, parmi tous les éléments pris en compte pour la restructuration des formations universitaires au Chili, les tendances internationales et la méthodologie du projet Tuning ${ }^{5}$ (né luimême en Europe) en ont fait partie. Dans ce sens, selon Contreras Sanzana et Villalobos Claveria (2010, p. 411), « les cours de formation des enseignants ont adopté les modèles politiques et sociaux de l'époque, les processus d'éducation et de formation étant les témoins de l'époque, contribuant au développement du pays sur la base d'un programme fonctionnaliste ». Dans l'air du temps de cette période, pour le cas du Chili, il y avait des orientations venant en grande partie de certains organismes internationaux, qui ont propagé dans le pays l'approche par compétences dans toutes les formations universitaires, y compris la formation des enseignants. 
La formation des enseignants au Chili a continué son évolution et, après un intense débat et un long processus participatif de consultation impliquant différents acteurs du système éducatif, la loi 20.903 a été promulguée en 2016. Cette loi crée un système de développement professionnel et de carrière pour les enseignants, en introduisant plusieurs éléments : quels sont ces éléments et quels changements introduisent-ils dans la formation des enseignants?

\section{Une nouvelle loi de la profession et la formation : régulation et consolidation de «l'État évaluateur »}

La loi 20.903, se voulant un outil de cadrage général pour la carrière enseignante, propose une mise en place progressive (2016-2026) de huit points essentiels : 1) la régulation de l'offre de formation et l'assurance d'un système formatif de haute qualité; 2) la régulation de l'entrée des étudiants aux parcours de pédagogie, à travers l'établissement d'un « minimum » en matière de résultats académiques permettant l'accès; 3) la régulation de la qualité des diplômés de pédagogie;4) la régulation de l'entrée à la profession enseignante; 5) l'induction ou l'accompagnement à l'initiation enseignante; 6) la création d'une carrière attractive; 7) des conditions de travail spécifiques; 8) une retraite consistante avec la carrière (Ruffinelli, 2016).

Concernant la régulation de l'offre de formation et l'assurance d'un système de formation de haute qualité, deux éléments essentiels sont retenus par la loi. Le premier concerne le type d'institution pouvant dispenser et offrir les programmes de formation du professorat. Contrairement au fonctionnement établi par la loi LOCE où des institutions à vocation plus technique pouvaient offrir des diplômes en enseignement (instituts de formation professionnelle, sans aucun lien avec la recherche fondamentale ou appliquée, par exemple), ce sont les universités qui peuvent dorénavant exclusivement former les enseignants. Le second élément essentiel de la loi en lien avec la formation des enseignants concerne l'obligation de soumettre à un processus d'accréditation ${ }^{6}$ chaque programme de formation à l'enseignement. Seuls les programmes d'odontologie, de médecine et maintenant ceux de l'enseignement sont contraints à passer par cet outil évaluatif. À la suite de l'évaluation, l'organisme évaluateur (Consejo Nacional de Acreditación - CNA) accorde entre 1 et 7 ans d'accréditation. Cette évaluation est réalisée sur base de différents critères regroupant : les exigences en matière d'infrastructure et d'équipement, les caractéristiques et de la qualité du corps enseignant, la présence de stages dès les premières années de formation et à caractère progressif (en objectifs et en niveau de difficulté), l'application de tests diagnostiques en milieu de parcours durant la formation initiale, l'existence de dispositifs de soutien au processus formatif des étudiants, l'exigence de cohérence entre l'itinéraire de formation et le profil professionnel en fin d'études, et l'existence de conventions avec des établissements scolaires qui assurent un lien plus solide et stable entre l'université et l'école.

D'un point de vue critique, Ruffinelli (2016) affirme que cette loi n'assure pas des orientations minimes communes afin d'assurer la cohésion entre les très nombreuses institutions de formation. En mettant en avant la liberté et l'autonomie des universités - comme pour le cas de la loi LOCE, influencée selon Beech (2007) par des organismes internationaux, la décentralisation et la dérégulation de la formation initiale des enseignants se voit prolongée. Parallèlement, il y aurait une prolongation de la tension entre les éléments définis par Darling-Hammond (2012) comme propres à une approche organique 
de la formation (c'est-à-dire à visée de développement professionnel intégral), et ceux relevant d'une approche de gestion et de management de la formation et de reddition de comptes (c'est-à-dire davantage orientée par une visée de maîtrise de compétences et de techniques d'enseignement); d'une part, les éléments 2, 5, 6, 7 et 8 de la loi (énoncés supra) s'inscrivent dans une approche organique, tandis que les éléments 1, 3 et 4 sont liés à l'approche de gestion. Finalement, c'est précisément dans le choix des régulations par l'évaluation à travers de tests diagnostiques et d'habilitation à enseigner (étudiants/futurs enseignants) et l'accréditation des institutions et des programmes de formation (à travers de la constitution de dossiers très lourds et des entrevues avec des acteurs institutionnels) que la logique de reddition de comptes s'enracine à nouveau et plus profondément. Selon Parcerisa et Falabella (2017, p. 4-5) :

La reddition de comptes est une pièce centrale dans les recommandations élaborées par les principaux organismes internationaux du champ éducatif, comme par exemple, l'Organisation pour la Coopération et le Développement Économique (OCDE) et la Banque Mondiale (BM). À ce propos, la reddition de comptes se considère centrale pour améliorer les résultats (OCDE, 2009) et la qualité des systèmes éducatifs, et pour aligner les incitatifs qui orientent l'action des différents acteurs scolaires (World Bank, 2015).

La singularité de l'usage chilien de la reddition de comptes a trait à la finalité de régulation du marché scolaire (choix des établissements scolaires par les élèves et leurs parents, et choix des organismes de formation par les futurs candidats à l'enseignement), en assumant de manière centrale le rôle d'un

«État évaluateur » (Maroy, 2009). Ce choix assumé amène l'État à définir des standards et mécanismes complexes d'évaluation et d'accréditation des institutions de formation des futurs enseignants, plutôt qu'à établir un consensus clair sur la formation des enseignants (organisation et modèles de formation, curriculum et contenus de formation).

\section{Le Chili, le bon élève : mimêsis d'un regard sur la formation des enseignants}

Comme cela a été discuté et documenté dans les sections précédentes, la formation des enseignants au Chili a été le témoin de transformations importantes dans les dernières décennies. Sous l'influence de certains organismes internationaux, la formation des enseignants a adopté et incarné de manière peu critique un modèle essentiellement basé sur une approche par compétences (par ailleurs définies de manière décentralisée et quelque part disparates par chaque centre de formation), s'inscrivant par ailleurs dans une tension entre les approches organiques (aspects professionnels) et de gestion du métier (aspects techniques) (Cochran-Smith et Lytle, 1999; Falabella, 2015).

Parallèlement, la régulation de la formation des enseignants s'est inscrite, depuis les années 1980, dans la lignée d'un État évaluateur (Parcerisa et Falabella, 2017) qui emploie une logique de reddition de comptes en matière de politiques éducatives, au travers de la mesure des performances et des résultats des acteurs (futurs enseignants/étudiants), des institutions et de leurs programmes. Ce phénomène fait du Chili l'un de systèmes éducatifs les plus centrés sur la performance et sur l'évaluation de la planète (Núñez-Moscoso et Flores Gonzalez, 2017). 
Ces deux aspects, l'adoption et l'incarnation exacerbée d'un modèle par compétences et d'une logique de reddition de comptes où l'évaluation des résultats est centrale, semblent dès lors s'inscrire dans le déploiement de la formation des enseignants au Chili, au moins dans les aspects évoqués, comme la mimêsis d'une politique transnationale.

\section{À titre de conclusion : potentialités et limites des politiques en matière de formation des enseignants}

Tout d'abord, il nous semble pertinent d'établir quelques nuances : l'influence internationale dans les politiques éducatives comporte la possibilité d'une prise de distance vis-à-vis les expériences nationales et de mettre ces dernières en perspective à la lumière d'expériences et expertises internationales, et dès lors mettre au profit d'un système éducatif l'expérience d'un autre système éducatif, d'identifier des ressources et outils qui peuvent, adaptation médiante, être repris et employés, parmi de multiples et très riches possibilités. Cependant, ceci ne peut être fait au détriment d'une « culture scolaire locale » et d'une réflexion critique quant à la mise en avant et l'adoption d'instruments dont les effets sur différents acteurs demeurent controversés. À notre avis, l'hyper évaluation comporte un risque d'épuisement de la capacité de travail (de formateurs et directifs qui montent les dossiers d'accréditation, par exemple) et de la capacité d'apprentissage chez les futurs enseignants (qui sont soumis à la pression du test qui diagnostique, mais aussi habilite aux fonctions d'enseignants).

Cela étant dit, les nouvelles conditions de travail des enseignants, les exigences d'entrée dans la formation, la volonté et l'énergie déployée en vue de l'amélioration de la formation du professorat portées par les évolutions récentes en matière de politiques éducatives chiliennes, sont des éléments extrêmement positifs. Cependant, lélément le plus significatif de la formation des enseignants reste la logique obstinée consistant à mettre en place des politiques mimétiques qui, telles que dans un rituel sacré, continuent à provoquer une « transe » acritique.

Précisément, nous pouvons remarquer l'absence d'une réflexion sur la posture et le rôle de l'État dans la définition d'un projet politique en éducation au sens large. Ici, la formation des enseignants n'est qu'une partie d'un tout plus complexe. Cependant :

une telle proposition implique un État politiquement actif comme régulateur et organisateur du système enseignant dans tout son sens et qui possède les attributions pour accomplir sa tâche. Ceci est un contresens dans un schéma de marché ou dans un modèle où l'État est subsidiaire, en conséquence on exige d'une manière quelque chose qui est au-delà d'un fait concret que l'on ne veut pas ou que l'on ne peut pas changer. (Donoso Diaz, 2008, p. 451)

Dans ce contexte, un soutien structurel à la profession enseignante demeure un élément clef pour penser et organiser la formation des enseignants, et ce, dans un système éducatif garant de lorganisation et de la cohérence d'un projet éducatif commun. Cependant, tout porte à croire qu'un rituel sacré de mimétisme politique trouve ici ses limites; en revanche, un « retour à l'essentiel » peut-être nécessaire, comme le préconise Ball (2015, p. 7) : 
Il est temps de revenir à l'essentiel - de réfléchir sérieusement à l'objectif de l'éducation, à ce que cela signifie d'être éduqué, à la raison d'être des écoles et, parallèlement et de manière cruciale, à qui devrait décider de ces choses. Une réflexion aussi approfondie doit aller au-delà des opinions des «experts» et des entrepreneurs politiques et de ceux qui ont des intérêts commerciaux dans l'éducation, pour entendre ce que les parents, les étudiants et les enseignants ont à dire sur ce que l'éducation devrait être à leur avis - « sur ce que l'éducation pourrait être, plutôt que sur ce qu'elle est devenue.

Ball semble nous avertir d'une manière claire, directe et critique que le présent et le futur de l'éducation ne sont pas ailleurs. Ce retour à l'essentiel est, dans toute sa profondeur, anti-mimêtique, et invite à un regard critique et fondamental :

Nous devons remettre en question la "logique de nécessité" qui a dominé la politique éducative du New Labour et de la Coalition, ainsi que celle de l'Union européenne, de la Banque mondiale, de l'Organisation de Coopération et de Développement Économiques et de l'UNESCO, articulée à travers leurs nombreux et répétitifs "textes persuasifs" qui mettent en évidence la subordination de la "politique sociale aux exigences de flexibilité du marché du travail et/ou d'employabilité et aux impératifs perçus de compétitivité structurelle ou systémique”. (Ball, 2015, p. 7)

Dans ce sens, il est nécessaire de nous rappeler qu'une formation pour les enseignants se doit d'être ancrée dans un contexte sociohistorique. Le fait que les systèmes éducatifs se reconnectent avec leur milieu constitue dès lors un point de départ nécessaire.

\section{Notes}

1 Dans ce travail, la notion de paradigme est entendue comme étant constituée « par un certain type de relations logiques, extrêmement fortes entre des notions maitresses, des notions clés, des principes clés » (Morin, 1990, p. 79). Dans ce sens, il s'agit d'un ensemble notionnel partagé qui oriente les discours, les visions du monde et l'action. Cette idée est appliquée aux politiques de formation des enseignants au Chili, en mettant à jour deux caractéristiques du paradigme : 1) une disjonction économétrique qui prône « un modèle caractérisé par une orientation professionnalisante, visant à former à l'élaboration et l'application des techniques liées étroitement aux notions d'efficience, d'efficacité, d'effectivité et de compétitivité » (Sánchez Carreño et Pérez Rodríguez, 2011, p. 143); 2) une disjonction disciplinaire, qui interpelle l'idée de connaissance dans laquelle l'erreur et l'illusion ne font pas partie de la connaissance à enseigner (Morin, 1999).

2 «Internationalisation » et " globalisation » sont deux notions qui comportent une spécificité. Cependant, dans le cadre de cet article, elles ne sont pas employées de manière théorique.

3 Par exemple, rappelons que les premiers établissements scolaires en Amérique latine ont été fondés par les jésuites, dans le cas du Chili plus précisément en 1595 (Contreras Gutierrez, 2014). De même, l’influence des mouvements pédagogiques européennes et leur apport au système de formation de maîtres est majeure.

4 Toutes les citations en version originale anglaise ou espagnole ont été traduites en français par l'auteur.

5 Le projet Tuning - Europe est une initiative internationale venant du processus Sorbonne-Bologne-PragueBerlin ayant pour objectif de lier et standardiser la formation supérieure dans l'espace européen en 1999, à travers l'internationalisation de compétences. La version Tuning-Amérique latine (proyecto Alfa Tuning América Latina) émerge en 2003 et est soutenue par la Commission Européenne et par un consortium d'universités du vieux continent, cordonnées par la Universidad de Deusto, Espagne (Muñoz, 2006) 
6 Rappelons qu'il existe aussi un processus d'accréditation institutionnelle : les universités sont évaluées par un organisme indépendant (Consejo Nacional de Acreditación - CNA), qui sanctionne la « qualité » de l'institution en matière de gestion institutionnelle et d'enseignement en premier cycle supérieur (obligatoire), mais aussi en enseignement de deuxième et troisième cycle supérieur, de recherche et lien avec le milieu social (en option). Il est important de savoir que le processus d'accréditation donne comme résultat entre 1 et 7 ans de validation. Après ce temps écoulé, un nouveau processus d'accréditation doit être réalisé.

\section{Références}

Ball, S. (1993). What is Policy? Texts, Trajectories and Toolboxes. Discourse, 13(2), 10-17.

Ball, S. (2015). Back to Basics: Repoliticising education. Forum, 51(1).

Barraza, A. (2007). La formación docente bajo una conceptualizacion comprehensiva y un enfoque por competencias. Estudios Pedagógicos, XXXIII(2), 131-153.

Beech, J. (2005). International agencies, educational discourse, and the reform of teacher education in Argentina and Brazil (19852002): A comparative analysis [Thesis]. Institute of Education, University of London.

Beech, J. (2007). La internacionalización de las políticas educativas en América Latina. Revista Pensamiento Educativo, 40(1), 153-173.

Bozal, V. (1987). Mimesis: Las imágenes y las cosas. Visor.

Canan, S. (2017). Influencia de los organismos internacionales en las politicas educacionales: ¿̇sólo hay intervención cuando hay consentimiento? CLACSO.

Cancino Cancino, V. et Iturra Herrera, C. (2018). Gestión Curricular en un Enfoque por Competencias: Aspectos Claves y Avances en el Sistema Universitario Chileno. En O. Leyva Cordero, F. Ganga Contreras, J. Tejada Fernández et A. Hernández $\mathrm{Paz}$ (Eds.), La formación por competencias en la educación superior: Alcances y limitaciones desde referentes de México, España y Chile (pp. 323-351). Tirant humanidades.

Cochran-Smith, M. et Lytle, S. L. (1999). Relationships of Knowledge and Practice: Teacher Learning in Communities. American Educational Research Association, 24, 249-305.

Contreras Gutierrez, A. (2014). La enseñanza jesuita en Chile colonial: Sus colegios, universidades y una aproximación a susmétodos y contenidos. Revista Historia de la Educación Latinoamericana, 16(22), 35-50.

Contreras Sanzana, G. et Villalobos Clavería, A. (2010). La formación de profesores en Chile: Una mirada a la profesionalización docente. Educación y Educadores, 13(3), 397-417.

Cox, C. et Gysling, J. (2009). La formación del profesorado en Chile, 1842-1987. Ediciones Universidad Diego Portales.

Darling-Hammond, L. (2012). Educar con calidad y equidad: Los dilemas del siglo XXI. Fundación Chile.

Donoso Diaz, S. (2008). El perfeccionamiento docente en Chile (1990-2007): ¿Estado versus mercado? Revista Brasileira de Educação, 13(39), 437-454.

Falabella, A. (2015). El mercado escolar en chile y el surgimiento de la nueva gestión pública: El tejido de la política entre la dictadura neoliberal y los gobiernos de la centroizquierda (1979 a 2009). Educação É Sociedade, 36(132), 699-722.

Fernández, V. (2008). Renovación curricular: Visión del programa MECESUP2. En CINDA (Ed.), Diseño curricular basado en competencias y aseguramiento de la calidad en la educación superior. (pp. 135-154).

Gutiérrez Canales, G. (2016). Sobre el concepto de mímesis en la antigua Grecia. Byzantion nea hellás, 35, 97-106. http:// dx.doi.org/10.4067/S0718-84712016000100005

Marcel, G. (1928). Journal métaphysique. Editions Gallimard.

Maroy, C. (2009). Convergences and hybridization of educational policies around 'postbureaucratic' models of regulation. Compare, 39(1), 71-84.

Morin, E. (1990). Introduction à la pensée complexe. ESF éditeur. 
Morin, E. (1999). Les sept savoirs nécessaires à l'éducation du futur. UNESCO.

Muñoz, A. L. (2006). Proyecto Tuning en Chile : Análisis de procesos de internacionalización de la educación superior. Calidad en la Educación, 249-271.

Núñez-Moscoso, J. et Flores Gonzalez, L. (2017). Travail enseignant et formation. Les politiques éducatives au prisme de la pensée complexe. En T. Piot et J.-F. Marcel (Eds.), Changements en éducation. Intentions politiques et travail enseignant (pp. 57-72). Octarès Editions.

Oliva, M. A. (2008). Politica educativa y profundizacion de la desigualdad en chile. XXXIV(2), 207-226.

Oreja Cerruti, M. et Vior, S. E. (2016). La educación y los Organismos Internacionales de crédito. Préstamos y recomendaciones para América Latina (2000-2015). Journal of Supranational Policies of Education, 4, 18-37.

Parcerisa, L. et Falabella, A. (2017). La Consolidación del Estado Evaluador a Través de Políticas de Rendición de Cuentas: Trayectoria, Producción y Tensiones en el Sistema Educativo Chileno. Education Policy Analysis Archives, 25(89), 1-27.

Pronko, M. (2000). As políticas de formação profissional impulsionadas pelos organismos internacionais no MERCOSUL. Um olhar sobre três casos (BID, OIT e UNESCO). En S. Yannoulas (Ed.), Atuais tendências na educação profissional (pp. 24-55). FLACSO/Brasil.

Ruffinelli, A. (2016). Ley de desarrollo profesional docente en Chile: De la precarización sistemática a los logros, avances y desafíos pendientes para la profesionalización. Estudios Pedagógicos, XLII(4), 261-279.

Sánchez Carreño, J. et Pérez Rodríguez, C. (2011). Hacia un currículo transdisciplinario: Una mirada desde el pensamiento complejo. Revista de Teoría y Didáctica de las Ciencias Sociales, 17, 143-164.

Tardif, M. et Desbiens, J.-F. (Eds.). (2014). La vogue des compétences dans la formation des enseignants. Bilan critique et perspectives d'avenir. Presses de l'Université Laval.

Valenzuela, J. M., Labarrera, P. et Rodriguez, P. (2008). Educación en Chile : Entre la continuidad y las rupturas. Principales hitos de las políticas educativas. Revista Iberoamericana de Educación, 48, 129-145.

Verger, A. et Parcerisa, L. (2018). Globalización, rendición de cuentas y gobernanza educativa: Análisis de un fenómeno en expansión. Con-Ciencia Social, 1, 59-73.

\section{Pour citer cet article}

Núñez-Moscoso, J. (2021). La formation des enseignants au Chili : est-elle une mimêsis de la politique (trans)national? Formation et profession, 29(3), 1-11. http://dx.doi.org/10.18162/fp.2021.657 